\title{
Cochlear implants in unilateral hearing loss for tinnitus suppression
}

\author{
Mohamed S Elgandy ${ }^{1,2 *}$ and Richard S Tyler ${ }^{2,3}$ \\ ${ }^{1}$ Department of Otolaryngology-Head and Neck Surgery, Zagazig University, Egypt \\ ${ }^{2}$ Department of Otolaryngology-Head and Neck Surgery, University of Iowa, Iowa City, Iowa \\ ${ }^{3}$ Department of Communication Sciences and Disorders, University of Iowa, Iowa City, USA
}

\begin{abstract}
Tinnitus is a pervasive symptom that can affect many people with hearing loss. It is found that its incidence is increasing due to accompanying occupational and environmental noise. Even, there is no standard treatment is present up till now, but cochlear implants (CI) positive effects are well proven and documented. Patients with unilateral severe hearing loss benefit from a cochlear implant; to improve their hearing and also often reduce the stress caused by their tinnitus. This chapter provides an overview of many publicly available reports about cochlear implant and tinnitus, including the mechanisms of tinnitus reduction by cochlear stimulation. We also review several articles demonstrating the benefit of cochlear implants for unilateral hearing loss and tinnitus. We believe that this approach will help many, and should be considered as standard practice and reimbursed.
\end{abstract}

\section{Introduction}

Unilateral hearing loss implies a profound sensori-neural hearing loss in one ear and no greater than a mild hearing loss in the opposite ear. Unilateral hearing loss affecting approximately 18.1 million persons in the United States [1]. Causes of unilateral hearing loss include infections (mumps, labyrinthitis and meningitis), ototoxicity, Meniere's disease, sudden idiopathic sensorineural hearing loss and temporal bone trauma. Unilateral hearing loss is accompanied by significant audiological consequences.

Patients with unilateral deafness frequently also experience tinnitus, which can have a profound impact on an individual's quality of life. Specifically, tinnitus has been associated with an increased incidence of depression, anxiety, hearing difficulties, difficult concentration and insomnia. In the U.S.A the FDA has not yet approved cochlear implantation in unilateral hearing loss. This chapter reviews some relevant literature and supports the notion of a CI for tinnitus in unilateral deafness [2].

\section{Tinnitus}

Tinnitus is the perception of a sound is when there is no external source it originates in the head: (it is not being overly sensitive to an external quiet sound) both the perception of the sound and reaction of patient to tinnitus should be considered [3].

It is helpful to differentiate between tinnitus which is problematic from that which does not. When the tinnitus occurs and how long an episode is? Or is the tinnitus present all over the day? [4].

Tinnitus classification is varible.it had been classified according to several facts, such as its site of generation and whether it is objective or subjective. Objective tinnitus is audible to someone other than the patient while subjective one is audible to patient alone. When tinnitus heard by physician, it is objective one which can be caused by middle ear pathology is spontaneous cochlear otoacoustic emissions so, using terms subjective and objective are not descriptive of tinnitus origin nor treatment. Therefor it had to be categorized according to its site of origin in a manner similar to sensory neural hearing loss. Regardless of the cause of tinnitus, the signal is eventually processed by the central auditory nervous system and consciously perceived in the auditory cortex.

\section{Prevalence}

The prevalence of troublesome tinnitus increases with age to 70 years $[5,6]$. Prevalence in men and women is similar. Prevalence in children is difficult to estimate, but results of available studies suggest that tinnitus experiences common. Children, however, seem less likely to be distressed by the perception [7].

\section{Psychological aspects of tinnitus}

Not all patients experience tinnitus in the same way, and intrinsic and extrinsic factors such as personality, psychosocial factors, and environment contribute to the patient's tinnitus reaction [8]. Some patients barely notice tinnitus, whereas others are severely affected by difficulty with concentration, sleep disturbances, anxiety, depression, or despair [9].

Sound carries meaning. Sound can evoke strong emotional reactions because of its importance to survival and because it is the primary medium for spoken language [10-12]. Our ears are always

${ }^{\star}$ Correspondence to: Mohamed Salah Elgandy, Department of OtolaryngologyHead and Neck Surgery, Zagazig University, Egypt, Tel: +20-55-2364612; E-mail: mohamedsalahibrahimsaleh-elgandy@uiowa.edu

Key words: unilateral hearing loss, tinnitus, electrical stimulation, cochlear implants

Received: November 12, 2018; Accepted: November 26, 2018; Published: November 30, 2018 
searching the world around us for meaningful or threatening sounds. Most sounds that have little meaning or are not interpreted as a threat are quickly habituated (not reacted to) by the central nervous system [13]. For example, sound of an electric fan. Unless an individual pay attention to hear the sound, the person is not aware that the sound is present, so there is a link between emotion and sound which makes the psychological upsets in some tinnitus patients [14]. In patients who typically seek treatment the tinnitus has likely become intrusive, annoying, or disturbing for them and may persist as a problem even though the underlying condition has been present for months or even years. The tinnitus can influence psychological state of the individual and the psychological reactions and negative associations can exacerbate the condition and, in effect, amplify the perception of tinnitus. Effective control of these maladaptive emotional reactions and beliefs is an important component of tinnitus management.

\section{Pathophysiology of tinnitus}

The study of tinnitus mechanisms is vitally important to develop effective treatments for tinnitus in all its forms and manifestations. There are three broad classifications of tinnitus [15], according to the anatomy and functional aspect of auditory pathway, which include conductive, sensorineural, and central tinnitus. Conductive tinnitus (Middle ear tinnitus) involves muscle twitching and blood vessels pulsing. This occurs, for example, in palatal myoclonus, high jugular bulb and glomus tympanicum.

Sensorineural tinnitus would have numerous subclasses, either generated from outer hair cells. Inner hair cells and auditory nerve. Central tinnitus would involve tinnitus originating anywhere in the central auditory pathways.

Many theories and models have been proposed to explain the pathophysiological basis of tinnitus [16-18]. The most prevalent theories involve hair cells, the auditory nerve, and the central auditory nervous system.

Although tinnitus activity is interpreted in the auditory cortex, Theories involving hair cells originate into cochlea from alterations of the spontaneous activity, including:

- An increase in rate.

- Decrease in rate

- Periodic activity

- Synchronous activity cross neurons

- Edge effect between active an inactive neuron [19].

With the loss of hair cells or hair cell function, afferent neurons appear to trigger aberrant auditory sensations at frequencies at or near the focus of the lesion [20]. This "edge effect" theory showed that tinnitus is usually associated with hearing loss, and explained why tinnitus frequency is usually related to frequencies involved in hearing loss, and why tinnitus could persists after the time expected for a normal recovery from noise exposure. It seems logic that tinnitus should be coded in the auditory temporal lobe, however it can have its own source any place in the auditory system and can be altered by other systems. The importance of the central nervous system in tinnitus had been proven for years [21]. Proof of central nervous system affection includes the following:

- When the vestibulocochlear nerve on the same side of tinnitus is cut, there is often no recorded change in tinnitus perception.
- When masking of tinnitus with noise in the same ear, patients sometimes can perceive tinnitus in the other ear.

- Unilateral tinnitus can be masked at comparable and at low levels in ipsilateral and contralateral ears.

Additional support for tinnitus of central origin has come from a series of animal studies that examined noise-induced hyperactivity in the dorsal cochlear nucleus (DCN). It was observed that the DCN exhibits a spontaneous activity pattern following noise exposure that is very similar to the activity induced by a low-level tone [22]. DCN hyperactivity also had been induced by the chemotherapeutic drug cisplatin [23]. The two findings suggest that DCN hyperactivity could be a neurophysiological correlation of noise- and cisplatin-induced tinnitus, although both noise and the cisplatin damaged the outer hair cells. In fact, that damage caused by cisplatin has been proven in association with DCN hyperactivity. Zacharek et al. [24] approached the question of whether DCN hyperactivity originates centrally or peripherally following exposure to intense noise. So, noise damaged cochlea was ablated, and DCN hyperactivity persisted. Thus, the central hyperactivity was not dependent on cochlear input, which documented that the hyperactivity could be originated centrally.

For tinnitus perception, special evaluation should be configured in the brain (auditory temporal area),

Such as:

1. Some neurons had high levels of spontaneous activity while others not so we should determine that.

2. Performing an internal histogram which is equivalent to periodic time intervals between action potential of different neurons.

3. Presence or absence of synchronous activity between neurons.

Cochlear activity can affect tinnitus wherever its coding develop .so despite tinnitus activity is configured in the auditory area, some cases of tinnitus can emerge from cochlea due to changes in spontaneous activity of cochlear hair cells .

\section{Treatments for tinnitus}

Tinnitus treatment presents a dilemma for clinicians and patients. The symptoms can be distressing but it is difficult to treat it if we do not understand the cause. How should a clinician approach tinnitus treatment? There are some situations with co-existent ear disease that may improve tinnitus if the underlying pathology is addressed (cholesteatoma, sudden sensorineural hearing loss, and ototoxicity). Identification of possible treatable pathology is an important reason for patients to see a physician for tinnitus. However for the vast majority of tinnitus sufferers have chronic, idiopathic tinnitus, for which there is no surgery or medications that has been approved.

Various drug regimens have been tried for tinnitus treatment. Occasionally there are encouraging reports in the literature but to the present, a drug treatment has not identified for treating tinnitus. Some bothersome tinnitus results in depression, anxiety, or other psychological disturbance. Some of these patients can benefit from pharmacotherapy. It is important to note that the therapy in these situations is for depression and anxiety not tinnitus. As with any bothersome, common disorder that lacks understanding and effective treatments, tinnitus is an easy target for scams [25]. Thousands of purported cures can be found on the internet and people waste billions of dollars on tinnitus treatment yearly. Some scams are blatant. Others are subtle. Honest, well-intentioned practitioners may believe in them. 
These unsubstantiated therapies may be most problematic overall. Each time thorough research finally identifies that a treatment is unfounded there will be more reluctance to accept a new therapy that may be effective.

\section{Cochlear implant and tinnitus}

At present, electric stimulation via a CI has proven to be a welltolerated and effective means of restoring hearing to over 400, 000 severely hearing-impaired individuals worldwide. However, using electric stimulation to suppress tinnitus has not been yet accepted worldwide. In the U.S.A it has not been yet approved by the Food and Drug Administration.

\section{Mechanisms of influencing tinnitus from the cochlea}

Tinnitus to be changed, the activity of auditory nerve fibers should be affected by electrical activity of cochlea through variety of mechanisms;

- Increasing activity

- Decreasing activity

- Interfering with periodic activity

- Desynchronizing activity across nerve neurons.

- Elimination of edge effect.

When we used an electrical periodic pulse train as a stimulus, some auditory neurons responded at certain frequencies of the stimulus [26]. It was documented by using at least at stimulus repitions $\bigotimes 3000$ pulses per second (PPS). Rubinstein et al. [27] viewed high rate stimulus $>(>3000$ PPS $)$ conditioner stimulus and found that it caused nerve driving at a rate beyond its ability to follow the electrical wave form , at that point activity cross neurons were more close to follow a Poisson process.

\section{When tinnitus develops due to decrease in spontaneous rate}

With electrical stimulation of the auditory nerve, the resting state of hair cells would be altered with subsequent increase in neuro mediators release and accompanying increase in auditory nerve activity, with activity close to normal neurons spontaneous rate, tinnitus could be decreased and relieved.

\section{When tinnitus develops as a result of Increase in spontaneous rate}

When tinnitus resulted from increase in spontaneous rate of hair cells at narrow region of cochlea, so by electrical stimulation, a larger adjacent array of neurons might decrease their tinnitus activity. Thus, by using continuous electrical stimulation, the hyperactive neurons might alter the abnormal neurons so they wouldn't be able to transmit activity. This reduction in the prominence could eliminate the tinnitus.

\section{If tinnitus results from a periodic activity in spontaneous rate}

When tinnitus caused by small number of neurons which exhibit periodic activity in their spontaneous rate, by electrical stimulation with noise, periodicity could be disrupted and this can be done by use of a conditioner stimulus which might be able to accomplish this without audibility of the stimulation.

\section{If tinnitus results from a synchronous activity in spontaneous rate across neurons}

Tinnitus can be also resulted from synchronous activity across many neurons so by using electrical stimulation, disruption of synchronous activity can occur. Using a noisy stimulation could interfere with synchronous activity but can create its random synchronous one, and then use of conditioner stimulus would be able to disrupt tinnitus without audibility of its synchronicity.

\section{When tinnitus develops due to "edge effect"}

Tinnitus cans results as an edge effect between normal hair cells with its own spontaneous activity and areas of hair cell loss without spontaneous activity. This edge effect might result from a loss of inhibition from the inactive region. Electrical stimulation and activation of neural responses of the inactive region might cause elimination of the edge and the origin of tinnitus might be discarded.

\section{Review of using electricity to reduce tinnitus \\ CI studies}

Patients who had CI for rehabilitation of their hearing loss and for good speech perception reported that their tinnitus reduced while speech processor is on, so here some studied which could discuss and document effect of $\mathrm{CI}$ on tinnitus perception.

- Some patients experience a total elimination of tinnitus after the CI surgery. Kim et al. [28] reviewed effect on tinnitus of the Nucleus multichannel CI in 15 patients; patients were distributed as follow (12 bilateral, one ipsilateral and two contralateral tinnitus). Evaluation of effect of ci on tinnitus was done in different situations: device on, device on with variable sounds and device off. When the implant is off, there was complete or partial relief from tinnitus in $85 \%$ of patients in the ipsilateral ear .when the implant was on, $77 \%$ of patients improved. When applying various sound stimuli $92 \%$ of patients showed partial or complete improvement and relief.

- Some patients report a reduction in their tinnitus only when the speech processor is turned on Tyler et al. [29] reported a study on 52 patients who were selected in six ci centers, the majority of them utilizing multichannel intra cochlear devices. They stated that among 42 patients who had preoperative tinnitus, $81 \%$ of them tinnitus was partially or totally disappeared, $17 \%$ tinnitus was stable remain unchanged and in $2 \%$ tinntius became worse after implantation.

- Some patients report a reduction in their tinnitus after the speech processor is turned off. Residual inhibition (RI) of tinnitus is the period of time in which a patient has a reduction of tinnitus perception following use of $\mathrm{CI}$ and after deactivation. Souliere et al. [30] described a study on 28 patients, and reported RI in 14 patients of 28 and he stated that 4 patients had an RI for $>2$ hours, 3 patients had an RI $<$ I hour, 3 patients had an RI of 30-60 minutes and 4 patients had an RI 5-10 minutes.

- Some patients with bilateral tinnitus report a reduction of tinnitus on both sides after receiving a unilateral CI [31] (Table 1).

\section{Extra cochlear Stimulation}

Kuk et al. [32] studied effect of using an electrode at surface of tympanic membrane to relief tinnitus They applied square, sine, and triangular waveforms at frequencies from 62 to $8000 \mathrm{~Hz}$. they first screened patients and noticed that tinnitus was changed .after that they 
Table 1. Studies on unilateral CI showing with results for tinnitus on both ears

\begin{tabular}{|c|c|c|}
\hline Study & Number of subjects & Results \\
\hline McKerrow et al. [36] & 6 & $67 \%$ reduced \\
\hline Souliere et al. [30] & 33 & $42 \%$ improved \\
\hline Hazell et al. [11] & 127 & $\begin{array}{l}52 \% \text { better } \\
47 \% \text { same } \\
1 \% \text { worse }\end{array}$ \\
\hline Kim et al. [28] & 15 & $\begin{array}{c}71 \% \text { improved } \\
4 \% \text { same }\end{array}$ \\
\hline Fukuda and Albernaz & 6 & $\begin{array}{c}20 \% \text { suppressec } \\
20 \% \text { decreased } \\
40 \% \text { same }\end{array}$ \\
\hline Demajumar et al. & 70 & $20 \%$ abolished \\
\hline
\end{tabular}

studied stimulation placebo effect on tinnitus perception but later on process was not allowed as patients were able to recognize either they were already stimulated or not.

They included 10 patients; five of them reported that tinnitus decreased in the initial then at the second phase they were asked to evaluate tinnitus loudness and to mask tinnitus acoustically in the other non-stimulated ear before, during and after 10 minute stimulation by electrical current.

This study was important as it used a psycho-acoustical measurement, the amount of masking required to mask the tinnitus to document the tinnitus reduction. $60 \%$ of patients reported tinnitus reduction without audibility of stimulus and the reduction was in the stimulated ear only in $75 \%$ of patients who had bilateral tinnitus. Three patients documented complete relief from tinnitus some patients stated that post implantation reduction lasted from 40 seconds to 4 hours.

Mielczarek et al. [33] used an active silver electrode in the ear canal filled with saline solution and a passive one surface electrode placed over the forehead to deliver pulsatile direct current stimulation. The idea for such electrode configuration was to allow passage of current flow directly across cochlea which sits in between the two electrodes. The treatment session involved 15 applications of electric stimulation over approximately 30 days, including 3-4 applications per week and each application was lasting about 4 minutes. $30 \%$ of patients in the experimental group reported relief of tinnitus immediately after electric stimulation, with $10 \%$ of them still having the relief of tinnitus perception after 90 days of stimulation. On the other hand, in the control group there were only $5-8 \%$ of the patients (without electrical stimulation) stated that their tinnitus was relieved.

Joos et al. [34] used two electrodes, one of them active over skin of temporal lobe and the other is passive on the other arm to deliver transcranial current for stimulation. They included 175 subjects in their study and found that marked tinnitus decrees was encountered by effect of direct stimulation starting at only $2 \mathrm{Ma}$ but due to deficiency of general direct current polarity which affect brain activity (excitatory and inhibitory) they supposed that results attributed to alteration of abnormal brain cortical activity in patients with tinnitus.

Perez et al. [35] placed a trans- tympanic needle electrode to the promontory in order to deliver charge balanced pulsatile stimulation in 10 patients who suffered from chronic tinnitus. This study was different from other studies in using three consecutive 30-minute stimulation sessions, every other day, and 7 of their 10 patients were having normal or mild hearing loss ( $40 \mathrm{~dB} \mathrm{HL}$ at $0.5,1,2$, and $4 \mathrm{kHz}$ ) in other studies patients had marked hearing loss. By comparison there was significant tinnitus reduction during or immediately after the multiple treatment sessions, and return to baseline after 4 weeks in $50 \%$ of the patients.

\section{Intra cochlear Stimulation}

McKerrow et al. [36] studied effect of using anon audible 2- to 6- $\mathrm{MHz}$ carrier and 30-minute broadband noise to stimulate the speech processor at a level which is comfortable to six patients who had CI, they reported that five patients out of six had rapid decrease to complete relief from their tinnitus and also tinnitus reduced bilaterally and that remain effective for about 15 minute. After that time tinnitus started to appear again .then a noise was presented through speech processor at a level which is comfortable for the patient and tinnitus was observed and reduced bilaterally. And that still effective for 20 minutes .by turning off the noise, tinnitus returned gradually in one ear and still absent in the other for 20 minutes and that agree with both Dauman et al. [37] and Dauman et al. [38].

Rubinstein et al. [39] viewed a study in which they used a conditioner stimulus of high frequency and tinnitus was suppressed in patients with CIs and with round window electrodes. Some subjects responded and tinnitus was relieved after several minutes of stimulation, but sometimes continuous stimulation became no effective once tinnitus suppression was achieved. Changes of both amplitude andlor repetition rate of the pulse train sometimes can reinstall tinnitus suppression.

\section{Electrical stimulation through implant (no external microphone)}

Arts et al. [40] reported that a CI can suppress tinnitus independent on environmental sounds by providing intra cochlear electrical stimulation and tinnitus can be relieved at least for minutes. The main goal of this study was to compare the long-term suppressive effects of looped (i.e. repeated) electrical stimulation (without environmental sound perception) with the standard stimulation pattern of a CI (with environmental sound perception), they concluded that no need for environmental sounds coding for tinnitus suppression with intra cochlear stimulation, therefore it is obviously cleared that tinnitus suppression by CI not only caused by shifting patient attention from tinnitus sound to environmental ones, and stated that use of both the standard clinical CI and the experimental Tinnitus Implant (TI) are for tinnitus treatment. These findings provide merits for a successful clinical application of the TI, especially in patients with residual hearing.

\section{CI in unilateral hearing loss but help with tinnitus}

Candidacy for CI continues to expand, but new innovations have been the application of CIs to subjects with unilateral hearing loss and sever tinnitus [41]. Previous studies had indicated that tinnitus in unilateral hearing loss can be severe, and refractory to treatment. It has also been approved that individuals using a CI in one ear and a hearing aid in the other are usually able to combine these two inputs to improve spatial hearing, and often to reduce troublesome tinnitus. There are a growing number of studies evaluating the effect of implantation for rehabilitation of the deficits associated with unilateral deafness over the past several years as more centers offer this treatment modality to patients with unilateral deafness. The vast majority report improvement in sound localization, speech understanding in quiet and noise, and in their tinnitus [41].

\section{CI in Unilateral Deafness for tinnitus}

Van de Heyning et al. [42] reviewed his first study in which CI used to treat patients with unilateral tinnitus in unilateral hearing loss. They 
included 22 adults in this study with unilateral severe tinnitus resulted from ipsilateral sensorineural hearing loss of variable etiologies. Nine of them were using a hearing aid in the other non-implanted ear. They measured tinnitus loudness with magnitude estimation (0-10), the Tinnitus Questionnaire was administered 1 month prior to surgery and $1,3,6,12,18$ and 24 months after the first fitting. After 24 months, this study reported complete tinnitus suppression in $14 \%$ of the participants and tinnitus improvement in $82 \%$ of the participants.

Kleinjung et al. [43] reported a case of an adult man with unilateral sensorineural hearing loss and ipsilateral severe tinnitus, tinnitus was reduced 1 month after implantation and completely disappeared 3 months postoperatively. Measurement of tinnitus severity was done by Tinnitus Questionnaire, VAS was used to measure tinnitus loudness and annoyance and Tinnitus Handicap Inventory (THI) to quantify the tinnitus-related handicap.

Palau et al. [44] investigated three subjects suffering from tinnitus who had cochlear implantation. Subject 1 and 3 used a standard CI and subject 2 used a CI with a noise habituator modulated via the audio input specifically designed for that study. By viewing THI and magnitude estimation after 6 months of implantation, tinnitus was suppressed completely in subject 1and decreased in both subjects 2,3. patients who was suffering from tinnitus for more than 20 years, showed the least tinnitus relief.

Buchner et al. [45] reported a study including five patients with sever to profound unilateral sensory neural hearing loss and ipsilateral severe tinnitus. Based on an average of four magnitude estimation scores, three patients had their tinnitus suppressed. Two of them reported a nearly complete tinnitus reduction. The remaining two of the study indicated that tinnitus could be reduced in certain situations. Here, tinnitus reoccurred due to psychological stress and nosier work environment.

Van de Heyning et al. [46] included 11 subjects with unilateral hearing loss, of which 10 were suffering from tinnitus. Based on magnitude estimation, at 6 months after CI activation, five of the subjects showed complete tinnitus suppression, and three showed improvement of their tinnitus. No tinnitus worsening was reported. By CI deactivated, tinnitus reoccurs to its initial strength. On the other hand, of the two participants in whom tinnitus did not change after implantation with the CI activated, one participant reported an increase in tinnitus severity when deactivation of the speech processor.

Jacob et al. [47] documented the effects of CI on the quality of hearing in unilateral hearing loss. They included 13 persons .11 of them suffered from tinnitus. They observed that cochlear implantation improved quality of hearing with superadded tinnitus relief in nine persons who stated that their tinnitus improved after CI without reporting of tinnitus worsening.

Ramos et al. [48] included 10 patients with unilateral hearing loss (sudden-onset hearing loss) and accompanying tinnitus in the ipsilateral ear, all patients were operated for CI. Similar T-levels and C-levels of the electrode responsible for the tinnitus pitch and the four collateral electrodes were used. Quantification of tinnitus handicap and loudness by THI as well as magnitude estimation, respectively, at 1 and 3 months postoperatively. Complete suppression of tinnitus was reported by two patients, seven patients reported decreased tinnitus handicap and loudness. no tinnitus worsening was observed. Interestingly, when CI was deactivated there was still improvement in tinnitus perception.

Kleine Punte et al. [49] published a study including 26 subjects with unilateral hearing loss undergoing CI surgery; all of them had unilateral severe-to-profound sensorineural hearing loss and severe tinnitus. Twenty-two of them had already reported by Van de Heyning et al. [42]. Based on magnitude estimates, 2 years after surgery, four subjects reported complete tinnitus relief. The others reported tinnitus improvement. In 24 subjects, tinnitus started to appear again at the same loudness after deactivation of CI. Two subjects showed no tinnitus one day after CI was deactivated. Four participants who were not described by Van de Heyning et al. [42], were observed up to one year post CI surgery. Comparing effect of tinnitus loudness between 22 persons described before, they stated that tinnitus loudness were stabilized 3-6 months postoperative and no difference encountered between tinnitus of narrow band, polyphonic tinnitus or pure tone tinnitus.

Zeng et al. [50] published a special design which is different from the others as they used experimental CI settings as an attempt to suppress tinnitus in one unilateral hearing loss participant. They noticed that, quality of hearing was not improved when they used an electrical stimulation pattern independent of environmental sounds and found also there was no tinnitus reduction observed with the standard clinical CI settings. Based on magnitude estimates, tinnitus was completely suppressed within 6 minutes using a certain stimulus but reoccurred within seconds to its original loudness after stopping intra cochlear electrical stimulation.

\section{Conclusions}

CIs have been a very successful device to help those with severe to profound hearing loss. Tinnitus can be very debilitating, and there is no cure. Nearly all tinnitus patients have a hearing loss. It is clear that many patients with hearing loss and tinnitus benefit, not only from improved hearing, but also from a reduction in their tinnitus. We believe that CIs should be used for treating tinnitus, at it should be reimbursed!

\section{References}

1. Lin FR, Niparko JK, Ferrucci L (2011) Hearing loss prevalence in the United States Arch Intern Med 171: 1851-1852. [Crossref]

2. Iwasaki S, Sano H, Nishio S, Takumi Y, Okamoto M, et al. (2013) Hearing handicap in adults with unilateral deafness and bilateral hearing loss. Otol Neurotol 34: 644-649. [Crossref]

3. Bentler RA, Tyler RS (1987) Tinnitus management. ASHA 29: 27-32. [Crossref]

4. Coles RR, Hallam RS (1987) Tinnitus and its management. Br Med Bull 43: 983-998. [Crossref]

5. Tyler RS, Babin RW (1986) Tinnitus. St. Louis: Mosby: 3201-3217.

6. Davis A, El Rafaie A (2000) Epidemiology of tinnitus. Tinnitus handbook. San Diego, CA: Singular, Thomson Learning: 1-23.

7. Baguley DM, McFerran DJ (1999) Tinnitus in childhood. Int J Pediatr Otorhinolaryngol 49: 99-105. [Crossref]

8. House JW, Brackmann DE (1981) Tinnitus: Surgical management. Tinnitus. Ciba Foundation Symposium London: Pitman. 85: 204-216.

9. Tyler RS, Baker LJ (1983) Difficulties experienced by tinnitus sufferers. J Speech Hear Disord 48: 150-154. [Crossref]

10. Hallam R, Rachman S, Hinchcliffe R (1984) Psychological aspects of tinnitus. Oxford, England: Pergamon Press. 3: 31-53.

11. Hazell JWP (1999) The TRT method in practice. Proceedings of the Sixth International Tinnitus Seminar, The Tinnitus and Hyperacusis Centre, London: 92-98.

12. Kandel ER (2000) From nerve cells to cognition: The internal cellular representation required for perception and action. Principles of neural science. Nework:McGraw-Hill: 381-403.

13. Domjan M, Burkhard B (1986) The principles of learning and behavior. Pacific Grove, CA: Brooks/Cole. 
14. Jastreboff PJ, Gray WC, Gold SL (1996) Neurophysiological approach to tinnitus patients. Am J Otol 17: 236-240. [Crossref]

15. Zenner HP, Pfister M (1999) Systematic classification of tinnitus. Proceedings of the Sixth International Tinnitus Seminar. International Tinnitus Seminar: 17-19.

16. Baguley DM (2002) Mechanisms of tinnitus. Br Med Bull 63: 195-212. [Crossref]

17. Eggermont JJ (2000) Physiological mechanisms and neural models. Tinnitus handbook. San Diego, CA: Singular: 85-122.

18. Møller AR (2003) Pathophysiology of tinnitus. Otolaryngol Clin North Am 36: 249266, v-vi. [Crossref]

19. Kiang NYS, Moxon EC, Levine RA (1970) Auditory-nerve activity in cats with normal and abnormal cochleas. Sensorineural Hearing Loss. Churchill Livingston; London, UK: $241-268$

20. Jastreboff PJ (1990) Phantom auditory perception (tinnitus): Mechanisms of generation and perception. Neurosci Res 8: 221-254. [Crossref]

21. Tyler RS (1981) Tinnitus. Tinnitus (CibaFoundation Symposium 85), London, UK: Pitman: 136-137.

22. Kaltenbach JA, Afman CE (2000) Hyperactivity in the dorsal cochlear nucleus after intense sound exposure and its resemblance to tone-evoked activity: A physiological model for tinnitus. Hear Res 140: 165-172. [Crossref]

23. Kaltenbach JA, Rachel JD, Mathog TA, Zhang J, Falzarano PR, et al. (2002) Cisplatininduced hyperactivity in the dorsal cochlear nucleus and its relation to outer hair cell loss: Relevance to tinnitus. J Neurophysiol 88: 699-714. [Crossref]

24. Zacharek MA, Kaltenbach JA, Mathog TA, Zhang J (2002) Effects of cochlear ablation on noise induced hyperactivity in the hamster dorsal cochlear nucleus: Implications for the origin of noise induced tinnitus. Hear Res 172: 137-143. [Crossref]

25. Nagler SM (2003) Tinnitus. A patient's perspective. Otolaryngol Clin North Am 36 235-238, v. [Crossref]

26. Abbas PJ (1993) Electrophysiology. Cochlear Implants: Audiological Foundations. Singular; San Diego, CA: 317-355.

27. Rubinstein JT, Tyler RS, Johnson A, Brown CJ (2003) Electrical suppression of tinnitus with high-rate pulse trains. Otol Neurotol 24: 478-485. [Crossref]

28. Kim HN, Shim YJ, Kim YM, Kim ES (1995) Effect of electrical stimulation on tinnitus in the profoundly deaf. Proceedings of the Fifth International Tinnitus Seminar. Portland, OR: American Tinnitus Association: 508-517.

29. Tyler RS, Kelsay D (1990) Advantages and disadvantages reported by some of the better cochlear-implant patients. Am J Otol 11: 282-289. [Crossref]

30. Souliere CR Jr, Kileny PR, Zwolan TA, Kemink JL (1992) Tinnitus Suppression following cochlear implantation. A multifactorial investigation. Arch Otolaryngol Head Neck Surg 118: 1291-1297. [Crossref]

31. Zwolan TA, Kileny PR, Souliere CR, Kemink JL (1992) Tinnitus suppression following cochlear implantation. Tinnitus91:proceedings of the Fourth International Tinnitus Seminar; Amsterdam, The Netherlands: Kugler: 423-426.

32. Kuk FK, Tyler RS, Rustad N, Harker LA, Tye-Murray N (1989) Alternating current at the eardrum for tinnitus reduction. J Speech Hear Res 32: 393-400. [Crossref]

33. Mielczarek M, Olszewski J (2014) Direct current stimulation of the ear in tinnitus treatment: a double-blind placebo-controlled study. Eur Arch Otorhinolaryngol 271: 1815-1822. [Crossref]
34. Joos K, De Ridder D, Van de Heyning P, Vanneste S (2014) Polarity specific suppression effects of transcranial direct current stimulation for tinnitus. Neural Plast: 930860.

35. Perez R, Shaul C, Vardi M, Muhanna N, Kileny PR, et al. (2015) Multiple electrostimulation treatments to the promontory for tinnitus. Otol Neurotol 36: 366372. [Crossref]

36. McKerrow WS, Schreiner CE, Snyder RL, Merzenich MM, Toner JG (1991) Tinnitus suppression by cochlear implants. Ann Otol Rhinol Laryngol 100: 552 558. [Crossref]

37. Dauman R, Tyler RS, Aran JM (1993) Intracochlear electrical tinnitus reduction. Acto Otolaryngol 113: 291-295. [Crossref]

38. Dauman R, Tyler RS (1993) Tinnitus suppression in cochlear implant users. $A d v$ Otorhinolaryngol 48: 168-173. [Crossref]

39. Rubinstein JT, Tyler RS (2004) Electrical suppression of tinnitus. Tinnitus: Theory and Management. BC Decker; Hamilton, Ontario, Canada: 326-335.

40. Arts RA, George EL, Griessner A, Zierhofer C, Stokroos RJ (2015) Tinnitus suppression by intracochlear electrical stimulation in single sided deafness-a prospective clinical trial: Part I. Audiol Neurootol 20: 294:313. [Crossref]

41. Baguley DM, Atlas MD (2007) Cochlear implants and tinnitus. Prog Brain Res 166 347-355. [Crossref]

42. Van de Heyning P, Vermeire K, Diebl M, Nopp P, Anderson I, et al. (2008) Incapacitating unilateral tinnitus in single-sided deafness treated by cochlear implantation. Ann Otol RhinolLaryngol 117: 645-652. [Crossref]

43. Kleinjung T, Steffens T, Strutz J, Langguth B (2009) Curing tinnitus with a Cochlear Implant in a patient with unilateral sudden deafness: a case report. Cases $J$ 2: 7462. [Crossref]

44. Masgoret Palau E, Merán Gil JL, Moreno Vidal C, Falcón González JC, Artiles Cabrera O, et al. (2010) Tinnitus and cochlear implantation. Preliminary experience. Acta Otorrinolaringol Esp 61: 405-411. [Crossref]

45. Buechner A, Brendel M, Lesinski-Schiedat A, Wenzel G, Frohne-Buechner C, et al (2010) Cochlear implantation in unilateral deaf subjects associated with ipsilateral tinnitus. Otol Neurotol 31: 1381-1385. [Crossref]

46. Arndt S, Aschendorff A, Laszig R, Prosse S, Laszig R, et al. (2010) Comparison of pseudo binaural hearing to real binaural hearing rehabilitation after cochlear implantation in patients with unilateral deafness and tinnitus. Otol Neurotol 32: 39-47.

47. Jacob R, Stelzig Y, Nopp P, Schleich P (2011) [Audiological results with cochlear implants for single-sided deafness]. HNO 59: 453-460. [Crossref]

48. Ramos Á, Polo R, Masgoret E, Artiles O, Lisner I, et al. (2012) Cochlear implant in patients with sudden unilateral sensorineural hearing loss and associated tinnitus Esp. Acta Otorrino laringol 63: 15-20. [Crossref]

49. Punte AK, Vermeire K, Hofkens A, De Bodt M, De Ridder D, et al. (2011) Cochlear implantation as a durable tinnitus treatment in single-sided deafness. Cochlear Implants Int 12: S26-S29. [Crossref]

50. Zeng FG, Tang Q, Dimitrijevic A, Starr A, Larky J, et al. (2011) Tinnitus suppression by low-rate electric stimulation and its electrophysiological mechanisms. Hear Res 277 : 61-66. [Crossref]

Copyright: (C2018 Elgandy MS. This is an open-access article distributed under the terms of the Creative Commons Attribution License, which permits unrestricted use, distribution, and reproduction in any medium, provided the original author and source are credited. 